\title{
Rotational excitation of symmetric-top molecular ions by electron impact
}

\author{
Shih-I Chu \\ Joint Institute for Laboratory Astrophysics, University of Colorado and National Bureau of Standards, Boulder, Colorado 80302
}

(Received 11 November 1974; revised manuscript received 2 April 1975)

\begin{abstract}
The theory for the rotational excitation of symmetric-top molecular ions by electron impact is formulated within the Coulomb-Born approximation. Analytical expressions are derived for the rotational transitions induced by dipole and by quadrupole interactions. The cross sections are large and finite at threshold and decrease with increasing electron-impact energy. The validity of the present theory for the description of lowenergy collisions is examined. Numerical calculations are reported for the excitation of the hydronium $\mathrm{H}_{3} \mathrm{O}^{+}$ ion.
\end{abstract}

\section{INTRODUCTION}

The excitation of molecular rotation is an important mechanism by which slow electrons lose energy in molecular gases. The efficiency with which the rotational levels of molecules are excited is a significant parameter in the cooling of dense ionized plasmas, in the interpretation of the process of radiative recombination, and in the determination of electron-velocity distributions in gaseous discharges, in electron-drift experiments, and in the ionospheres of the planets.

The rotational excitation of neutral molecules by electron impact has been widely investigated both theoretically and experimentally. The corresponding excitations of molecular ions are, however, more difficult to study experimentally and only a few theoretical treatments have ever been reported. Stabler ${ }^{1}$ has studied the rotational excitation of homonuclear molecular ions using a lowenergy expansion of the Coulomb-Born approximation. His work was extended by Sampson ${ }^{2}$ to include the effect of the long-range polarization interaction. The results of Stabler ${ }^{1}$ for the scattering of electrons by $\mathrm{H}_{2}{ }^{+}$are in satisfactory agreement with the more sophisticated fixed-nuclei calculations of Chang and Temkin. ${ }^{3}$ This demonstrates the validity of using the Coulomb-Born approximation for the description of the scattering of electrons by molecular ions. Stabler's theory, while providing an adequate treatment of the rotational excitation of quadrupolar molecular ions, is not applicable to the case of dipolar molecular ions. A more refined Coulomb-Born formulation was presented independently by Boikova and Obeydkov $^{4}$ and recently by Chu and Dalgarno, ${ }^{5}$ who extended the theory of Coulomb excitation of nuclei to the rotational excitation of linear-dipole molecular ions.

In this paper, we extend the new formulation to the case of rotational excitation of symmetric-top molecular ions and we apply the theory to the cal- culation of the cross sections for excitation of the hydronium ion $\mathrm{H}_{3} \mathrm{O}^{+}$. The results should be useful in the quantitative interpretation of the process of dissociative recombination of the $\mathrm{H}_{3} \mathrm{O}^{+}$ion, which is of great experimental interest.

\section{THEORY}

We consider the process

$$
e^{-}+X Y_{3}^{+}(J K M) \rightarrow e^{-}+X Y_{3}^{+}\left(J^{\prime} K^{\prime} M^{\prime}\right),
$$

where $J$ and $M$ are, respectively, the total angular momentum and its space-fixed $z$ component, and $K$ denotes the angular momentum around the symmetry axis $(|K| \leqslant J)$ of the symmetric-top molecule. We assume that the molecular ion $X Y_{3}^{+}$can be described as a symmetric-top rigid rotator having a permanent dipole moment $\mu$ in the direction of the symmetry axis of the molecule.

The long-range interaction between the symmetric-top molecular ion and the electron can be represented in the form

$$
V(r, \chi, \psi)=-\frac{1}{r}+\sum_{\lambda \nu} v_{\lambda \nu}(r) Y_{\lambda \nu}(\chi, \psi),
$$

where $(\chi, \psi)$ are the polar and azimuthal angles specifying the direction of the incident electron with respect to the molecule-fixed coordinates (Fig. 1). The potential $v_{\lambda \nu}(r)$ can be expressed in terms of the multipole-moment tensors ${ }^{6}$ $M_{x^{k y} \nu-k_{z} \lambda-\nu}^{(\lambda)}$ as follows ${ }^{7}$ :

$$
\begin{aligned}
v_{\lambda \nu}(r)= & -\frac{1}{r^{\lambda+1}}\left(\frac{4 \pi}{2 \lambda+1}\right)^{1 / 2} \frac{\lambda !}{[(\lambda+\nu) !(\lambda-\nu) !]^{1 / 2}}(-1)^{\nu} \\
& \times \sum_{k=0}^{\nu}(-i)^{\nu-k}\left(\begin{array}{l}
\nu \\
k
\end{array}\right) M_{x^{k} \nu \nu-k_{z} \lambda-\nu}^{(\lambda)}
\end{aligned}
$$

where

$$
\begin{gathered}
M_{x^{k} y^{\nu-k_{z} \lambda-\nu}}^{(\lambda)} \equiv \frac{(-1)^{\lambda}}{\lambda !} \int \rho\left(\overrightarrow{\mathrm{r}}^{\prime}\right) r^{\prime 2 \lambda+1} \frac{\partial^{\lambda}}{\partial x^{\prime k} \partial y^{\prime \nu-k} \partial z^{\prime \lambda-\nu}} \\
\times\left(\frac{1}{r^{\prime}}\right) d \overrightarrow{\mathbf{r}}^{\prime}
\end{gathered}
$$




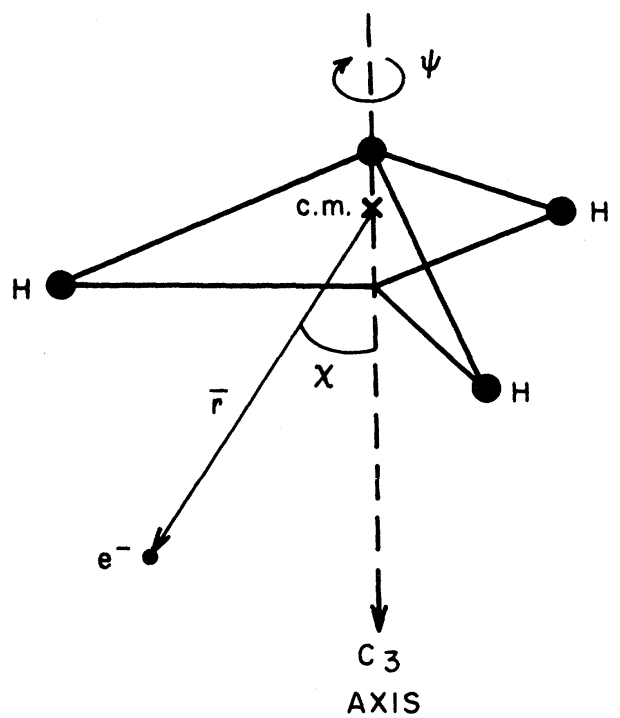

FIG. 1. Coordinate system used for electron scattering by the $\mathrm{H}_{3} \mathrm{O}^{+}$ion.

and $\rho$ is the charge distribution of the molecule. Since the symmetric-top molecular ion $X Y_{3}^{+}$possess $C_{3 v}$ symmetry, the term $v_{\lambda \nu}$ vanishes unless $|\nu|=3 n(n=0,1,2, \ldots)$. Thus, the leading nonvanishing terms of $v_{\lambda \nu}(r)$ are $^{7}$

$$
\begin{aligned}
v_{10}(r) & =-\left(\frac{4}{3} \pi\right)^{1 / 2}\left(1 / r^{2}\right) M_{z}^{(1)} \\
v_{20}(r)= & -\left(\frac{4}{5} \pi\right)^{1 / 2}\left(1 / r^{3}\right) M_{z z}^{(2)} \\
v_{30}(r) & =-\left(\frac{4}{7} \pi\right)^{1 / 2}\left(1 / r^{4}\right) M_{z z z}^{(3)} \\
v_{33}(r) & =v_{3,-3}(r) \\
= & -\left(\frac{36}{1260} \pi\right)^{1 / 2}\left(1 / r^{4}\right) \\
& \times\left(i M_{y y y}^{(3)}-3 M_{x y y}^{(3)}-3 i M_{x x y}^{(3)}+M_{x x x}^{(3)}\right) .
\end{aligned}
$$

If we choose the molecule-fixed $z$ axis to be the symmetry axis, then the three multipole-moment components $M_{z}^{(1)}, M_{z z}^{(2)}$, and $M_{z z z}^{(3)}$ can be identified with the dipole moment $\mu$, the quadrupole moment $Q$, and the octupole moment $\Omega$ of the molecular ion, respectively.

Transforming the coordinate system from the molecule-fixed frame to the space-fixed (SF) frame, we get ${ }^{8}$

$$
Y_{\lambda \nu}(\chi, \psi)=\sum_{\alpha} D_{-\alpha-\nu}^{(\lambda)}(\alpha \beta \gamma) Y_{\lambda_{Q}}(\theta, \phi),
$$

where $D^{(\lambda)}$ is the matrix element of the finite rotation operator, $(\alpha \beta \gamma)$ are the Euler angles.specifying the orientation of the symmetric top with respect to the frame of SF coordinate, and $(\theta, \phi)$ denote the direction of the electron with respect to the SF frame. With the use of this relation, the interaction potential between electron and molecular ion in the space-fixed frame becomes

$$
V(r, \theta, \phi)=-\frac{1}{r}+\sum_{\lambda \nu_{q}} v_{\lambda \nu}(r) D_{-\alpha-\nu}^{(\lambda)}(\alpha \beta \gamma) Y_{\lambda_{q}}(\theta, \phi) .
$$

We write the total Hamiltonian in atomic units in the form

$$
H=H_{0}+H^{\prime},
$$

where

$$
\begin{aligned}
& H_{0}=H_{\text {ion }}-\frac{1}{2} \nabla_{r}^{2}-1 / \gamma, \\
& H^{\prime}=+\sum_{\lambda=1}^{\infty} \sum_{\nu=-\lambda}^{\lambda} \sum_{q=-\lambda}^{\lambda} v_{\lambda \nu}(\gamma) D_{-q-\nu}^{(\lambda)}(\alpha \beta \gamma) Y_{\lambda_{q}}(\theta \phi),
\end{aligned}
$$

and $H_{\text {ion }}$ is the Hamiltonian for the isolated molecular ion. The unperturbed wave functions $\Psi^{(0)}$ separate into a Coulomb wave function $\Psi_{c}(\vec{k}, \vec{r})$ $\equiv|\overrightarrow{\mathrm{k}}\rangle$ and a symmetric-top rotator eigenfunction $\Psi_{J K M}$ defined by

$$
\Psi_{J K M}=\left[(2 J+1) / 8 \pi^{2}\right]^{1 / 2} D_{M K}^{(J)}(\alpha \beta \gamma) .
$$

The Coulomb-Born formula for the transition $(J K) \rightarrow\left(J^{\prime} K^{\prime}\right)$ is given by

$$
\begin{aligned}
& \sigma\left(J K \rightarrow J^{\prime} K^{\prime}\right) \\
& \quad=\frac{1}{4 \pi^{2}} \frac{k_{f}}{k_{i}} \sum_{M, M^{\prime}} \frac{1}{2 J+1} \int\left|\left\langle\Psi_{f}^{(0)}, H^{\prime} \Psi_{i}^{(0)}\right\rangle\right|^{2} d \Omega_{f},
\end{aligned}
$$

where $\overrightarrow{\mathrm{k}}_{i}$ and $\overrightarrow{\mathrm{k}}_{f}$ are the initial and final wave vectors of the scattered electron and $\Omega_{f} \equiv \hat{k}_{f}$ is the unit vector along $\overrightarrow{\mathrm{k}}_{f}$. To evaluate the integral of Eq. (12), consider the transition matrix element

$$
\begin{aligned}
A_{f i} & \equiv\left\langle\Psi_{f}^{(0)}, H^{\prime} \Psi_{i}^{(0)}\right\rangle \\
& =\left\langle\Psi_{J^{\prime} K^{\prime} M^{\prime}}(\alpha \beta \gamma)|I(\alpha \beta \gamma)| \Psi_{J K M}(\alpha \beta \gamma)\right\rangle,
\end{aligned}
$$

where

$$
\begin{aligned}
I(\alpha \beta \gamma) & \equiv\left\langle\overrightarrow{\mathrm{k}}_{f}\left|H^{\prime}\right| \overrightarrow{\mathrm{k}}_{i}\right\rangle \\
& =\sum_{\lambda \nu_{q}} D_{-\alpha-\nu}^{(\lambda)}(\alpha \beta \gamma)\left\langle\overrightarrow{\mathrm{k}}_{f}\left|v_{\lambda \nu}(\gamma) Y_{\lambda \boldsymbol{q}}(\theta \phi)\right| \overrightarrow{\mathrm{k}}_{\boldsymbol{i}}\right\rangle .
\end{aligned}
$$

Rewrite Eq. (3) as

$$
v_{\lambda \nu}(r)=-\left(1 / r^{\lambda+1}\right) M_{\lambda \nu}
$$

where $M_{\lambda \nu}$ is a constant (real or complex) independent of $r$. Substituting (15) into (14), we get

$I(\alpha \beta \gamma)=-\sum_{\lambda \nu_{\boldsymbol{q}}} M_{\lambda \nu} D_{-\boldsymbol{q}-\nu}^{(\lambda)}(\alpha \beta \gamma)\left\langle\overrightarrow{\mathrm{k}}_{f}\left|r^{-\lambda-1} Y_{\lambda_{\boldsymbol{q}}}(\theta \phi)\right| \overrightarrow{\mathrm{k}}_{i}\right\rangle$.

To calculate the integral in $\left(14^{\prime}\right)$, we expand the Coulomb wave functions into partial waves, $, 4,9$ 


$$
\begin{aligned}
\left|\overrightarrow{\mathrm{k}}_{i}\right\rangle=\sum_{l_{i} m_{i}} 4 \pi(-1)^{m_{i}} i^{l_{i}} e^{i \sigma_{l_{i}}\left(\eta_{i}\right)} & \\
& \times Y_{l_{i},-m_{i}}\left(\hat{k}_{i}\right) Y_{l_{i} m_{i}}(\hat{\gamma})\left(k_{i} r\right)^{-1} F_{l_{i}}\left(k_{i} r\right)
\end{aligned}
$$

and

$$
\begin{aligned}
\left|\overrightarrow{\mathrm{k}}_{f}\right\rangle=\sum_{l_{f} m_{f}} 4 \pi(-1)^{m_{f}} i^{l_{f}} e^{-i \sigma_{l_{f}}\left(\eta_{f}\right)} & \\
& \times Y_{l_{f},-m_{f}}\left(\hat{k}_{f}\right) Y_{l_{f} m_{f}}(\hat{\gamma})\left(k_{f} \gamma\right)^{-1} F_{l_{f}}\left(k_{f} \gamma\right),
\end{aligned}
$$

where $\sigma_{l}(\eta)=\arg \Gamma(l+1+i \eta)$ is the Coulomb phase shift $\eta=-1 / k$ and $F_{l}(k r)$ is the regular solution to the radial equation for orbital angular momentum $l, 9$

$$
\begin{aligned}
F_{l}(k r)= & e^{-\pi \eta / 2} \frac{|\Gamma(l+1+i \eta)|}{2 \Gamma(2 l+2)}(2 k r)^{l+1} e^{-i k r} \\
& \times_{1} F_{1}(l+1-i \eta, 2 l+2 ; 2 i k r) .
\end{aligned}
$$

Thus, ${ }^{4 \cdot 5}$

$$
\begin{aligned}
& \left\langle\overrightarrow{\mathrm{k}}_{f}\left|\boldsymbol{r}^{-\lambda-1} Y_{\lambda_{\boldsymbol{q}}}\right| \overrightarrow{\mathrm{k}}_{i}\right\rangle=(4 \pi)^{3 / 2} \sum_{l_{i} l_{f} m_{i} m_{f}} i^{l_{i}-l_{f}}(-1)^{\alpha} e^{i\left(\sigma_{l_{i}}+\sigma_{l_{f}}\right)}\left[\left(2 l_{i}+1\right)\left(2 l_{f}+1\right)(2 \lambda+1)\right]^{1 / 2} Y_{l_{i},-m_{i}}\left(\hat{k}_{i}\right) Y_{l_{f} m_{f}}\left(\hat{k}_{f}\right) M_{l_{i} l_{f}}^{*-1}, \\
& \times\left(\begin{array}{lll}
l_{i} & l_{f} & \lambda \\
0 & 0 & 0
\end{array}\right)\left(\begin{array}{ccc}
l_{i} & l_{f} & \lambda \\
m_{i} & -m_{f} & q
\end{array}\right)
\end{aligned}
$$

where the radial matrix element $M$ is defined by

$$
M_{l_{i} l_{f}}^{-\lambda-1} \equiv \frac{1}{k_{i} k_{f}} \int_{0}^{\infty} F_{l_{f}}\left(k_{f} r\right) r^{-\lambda-1} F_{l_{i}}\left(k_{i} r\right) d r .
$$

Hence,

$$
I(\alpha \beta \gamma)=-\sum_{\lambda \nu q l_{i} l_{f} m_{i} m_{f}} M_{\lambda \nu} T_{l_{i} m_{i} l_{f} m_{f}}^{\lambda_{q}} D_{-q-\nu}^{(\lambda)}(\alpha \beta \gamma) Y_{l_{\boldsymbol{i}}-m_{\boldsymbol{i}}}\left(\hat{k}_{\boldsymbol{i}}\right) Y_{l_{f} m_{f}}\left(\hat{k}_{f}\right) M_{l_{\boldsymbol{i}} l_{f}}^{-\lambda-1},
$$

where

$$
T_{l_{i} m_{i} l_{f} m_{f}}^{\lambda}=(4 \pi)^{3 / 2}(-1)^{\alpha} e^{i\left(\sigma_{l_{i}}+\sigma_{\left.l_{f}\right)} i^{l_{i}-l_{f}}\left[\left(2 l_{i}+1\right)\left(2 l_{f}+1\right)(2 \lambda+1)\right]^{1 / 2}\right.}\left(\begin{array}{ccc}
l_{i} & l_{f} & \lambda \\
0 & 0 & 0
\end{array}\right)\left(\begin{array}{ccc}
l_{i} & l_{f} & \lambda \\
m_{i} & -m_{f} & q
\end{array}\right) .
$$

The transition matrix element $A_{f i}$ can now be evaluated in the form

$$
A_{f i}=\left\langle\Psi_{J^{\prime} K^{\prime} M^{\prime}}|I(\alpha \beta \gamma)| \Psi_{J K M}\right\rangle=\sum_{\lambda \nu_{q} l_{i} l_{f}} G_{l_{i} l_{f} J J^{\prime} M M^{\prime} K K^{\prime}}^{\lambda_{q} \nu} T_{l_{f} q}\left(\hat{k}_{f}\right) M_{l_{i} l_{f}}^{-\lambda-1},
$$

where

$$
G_{i_{i} l_{f} J J^{\prime} M M^{\prime} K K^{\prime}}^{\lambda a \nu}=-M_{\lambda \nu} T_{l_{i}{ }^{0} l_{f} a}^{\lambda \alpha}\left[\left(2 l_{i}+1\right)(2 J+1)\left(2 J^{\prime}+1\right) / 4 \pi\right]^{1 / 2}(-1)^{M^{\prime}-K^{\prime}}\left(\begin{array}{ccc}
J & J^{\prime} & \lambda \\
M-M^{\prime} & -q
\end{array}\right)\left(\begin{array}{ccc}
J & J^{\prime} & \lambda \\
K & -K^{\prime} & -\nu
\end{array}\right) \cdot
$$

Note that in deriving Eq. (23), we have taken the space-fixed $z$ axis along $\vec{k}_{i}$ and we have used the formula

$$
\frac{1}{8 \pi^{2}} \int d \Omega D_{m_{1}^{\prime} m_{1}}^{\left(j_{1}\right)}(\Omega) D_{m_{2}^{\prime} m_{2}}^{\left(j_{2}\right)}(\Omega) D_{m_{3}^{\prime} m_{3}}^{\left(j_{3}\right)}(\Omega)=\left(\begin{array}{ccc}
j_{1} & j_{2} & j_{3} \\
m_{i}^{\prime} & m_{2}^{\prime} & m_{3}^{\prime}
\end{array}\right)\left(\begin{array}{lll}
j_{1} & j_{2} & j_{3} \\
m_{1} & m_{2} & m_{3}
\end{array}\right)
$$

where $\Omega=(\alpha \beta \gamma)$ and $d \Omega=d \alpha \sin \beta d \beta d \gamma$. Substituting Eq. (22) into Eq. (12), and carrying through the integra tion and summations, we get finally

$$
\sigma\left(J K \rightarrow J^{\prime} K^{\prime}\right)=\sum_{\lambda=1}^{\infty} \sigma_{\lambda, \nu=K-K^{\prime}}\left(J K \rightarrow J^{\prime} K^{\prime}\right),
$$

where

$$
\sigma_{\lambda \nu}\left(J K \rightarrow J^{\prime} K^{\prime}\right)=4\left(\frac{k_{f}}{k_{i}}\right)\left(M_{\lambda \nu}\right)^{2}\left(2 J^{\prime}+1\right)\left(\begin{array}{ccc}
J & J^{\prime} & \lambda \\
K & -K^{\prime} & -\nu
\end{array}\right)^{2} \sum_{l_{i} l_{f}}\left(2 l_{i}+1\right)\left(2 l_{f}+1\right)\left(\begin{array}{ccc}
l_{i} & l_{f} & \lambda \\
0 & 0 & 0
\end{array}\right)^{2}\left|M_{l_{i} l_{f}}^{\lambda-1}\right|^{2} .
$$

Because of the properties of the $3 j$ symbol

$$
\left(\begin{array}{ccc}
J & J^{\prime} & \lambda \\
K & -K^{\prime} & -\nu
\end{array}\right)
$$


only the terms $\sigma_{\lambda \nu}$ with $(\lambda, \nu)$ satisfying the conditions

$$
\left|J-J^{\prime}\right| \leqslant \lambda \leqslant J+J^{\prime}
$$

and

$$
K-K^{\prime}=\nu
$$

contribute to the transition $(J K)-\left(J^{\prime} K^{\prime}\right)$. In the special case when both $\nu$ and $K$ are zero, there is the additional parity rule that $J+J^{\prime}+\lambda$ is an even integer, and Eq. (25) reduces to the expression for the excitation of linear rigid-rotor ions. ${ }^{5}$ In the latter case, the transition $|\Delta J|=1$ is caused by the odd terms with $\lambda=1,3,5, \ldots$ and not by the even terms. The even terms with $\lambda=2,4,6, \ldots$ cause the transition $|\Delta J|=2$. When $K \neq 0$, however, the selection rules (26a) and (26b) hold, but the parity rule need not be satisfied even when $\nu=0$. And all the terms satisfying (26a) and (26b) contribute to the transition $J \rightarrow J^{\prime}$. For example, any term with $\lambda \geqslant 1$ may induce the transition $|\Delta J|=1$ in the case of $K \neq 0$.

\section{ROTATIONAL EXCITATION INDUCED BY DIPOLAR INTERACTION}

The leading term which contributes to the lowenergy scattering of electrons from polar symmetric-top molecular ions is the electron-permanent dipole interaction. In this case $\lambda=1, \nu=0$, $M_{10}=-\left(\frac{4}{3} \pi\right)^{1 / 2} \mu$, and

$$
\begin{aligned}
\sigma_{10}\left(J K \rightarrow J^{\prime}=J \pm 1, K^{\prime}\right)= & \delta\left(K, K^{\prime}\right)\left(\frac{3}{4 \pi^{2}}\right)\left(\frac{\pi}{k_{i}^{2}}\right) \mu^{2}\left(2 J^{\prime}+1\right) \\
& \times\left(\begin{array}{ccc}
J & J^{\prime} & 1 \\
K & -K & 0
\end{array}\right)^{2} f_{E 1}\left(\eta_{i}, \xi\right),
\end{aligned}
$$

where $\xi=\eta_{f}-\eta_{i}$ and $f_{E 1}$ is a function related to the $E 1$ nuclear Coulomb excitation ${ }^{9}$ that can be evaluated by the following formula ${ }^{5}$ :

$$
\begin{aligned}
f_{E 1}\left(\eta_{i}, \xi\right)= & -\frac{32}{9} \pi^{3} \frac{\eta_{i} \eta_{f}}{\xi} \frac{1}{e^{2 \pi \xi}-1} \\
& \times \operatorname{Im}\left\{\frac{1}{\eta_{i}} F\left(i \eta_{i}, i \eta_{i}, 1-i \xi ; \frac{1}{\chi_{0}}\right)\left[F\left(1-i \eta_{i},-i \eta_{i}, 1+i \xi ; \frac{1}{\chi_{0}}\right)+e^{i \phi} F\left(1-i \eta_{f},-i \eta_{f}, 1-i \xi ; \frac{1}{\chi_{0}}\right)\right]+\eta_{i} \mp \eta_{f}\right\},
\end{aligned}
$$

where

$$
f_{E 1}=32 \pi^{3} / 9 \sqrt{3}
$$

$$
\phi=2 \arg \left[\Gamma(i \xi) \Gamma\left(i \eta_{i}\right) / \Gamma\left(i \eta_{f}\right)\right]+\xi \ln \left|\chi_{0}\right|,
$$

and

$$
\chi_{0}=-4 \eta_{i} \eta_{f} / \xi^{2}
$$

and

$$
\sigma_{10}\left(J K \rightarrow J^{\prime}=J \pm 1, K^{\prime}\right)=\delta\left(K, K^{\prime}\right)(8 \pi / 3 \sqrt{3})\left(\pi / k_{i}^{2}\right) \mu^{2} g_{ \pm} .
$$

Equation (27) may be rewritten more explicitly as $\sigma_{10}\left(J K \rightarrow J^{\prime}=J \pm 1, K^{\prime}\right)=\delta\left(K, K^{\prime}\right)\left(3 / 4 \pi^{2}\right)\left(\pi / k_{i}^{2}\right) \mu^{2} f_{E 1} g_{ \pm}$,

with

$$
g_{+}=\frac{(J+K+1)(J-K+1)}{(J+1)(2 J+1)} \text { if } J^{\prime}-J=+1
$$

and

$$
g_{-}=\frac{J^{2}-K^{2}}{J(2 J+1)} \text { if } J^{\prime}-J=-1 .
$$

It is interesting to note that because of the factor $\delta\left(K, K^{\prime}\right)$, the dipolar interaction cannot induce rotation around the symmetric axis.

The threshold behavior of the rotational excitation cross section is of special physical interest. In this case, ${ }^{5}$
Thus, the present theory predicts large and finite cross sections which vary as $k_{i}^{-2}$ near the rotational excitation threshold, in accord with the Wigner threshold law. ${ }^{10}$ In contrast, the planewave Born approximation predicts zero cross section at threshold. ${ }^{5}$ The Coulomb-Born approximation used by Stabler gives vanishing dipolar cross sections $\left(\sigma_{10}\right)$ for all energies considered (cf. Appendix). Thus, Eq. (30) appears to be the first theoretical expression to predict correctly the threshold behavior for the rotational excitation processes induced by dipolar interaction.

\section{ROTATIONAL EXCITATION INDUCED BY QUADRUPOLAR INTERACTION}

For the case of quadrupolar interaction, we have $\lambda=2, \nu=0, M_{20}=-\left(\frac{4}{5} \pi\right)^{1 / 2} Q$, and

$$
\sigma_{20}\left(J K-J^{\prime} K^{\prime}\right)=\delta\left(K, K^{\prime}\right) \frac{16}{5} \pi Q^{2}\left(\frac{k_{f}}{k_{i}}\right)\left(2 J^{\prime}+1\right)\left(\begin{array}{ccc}
J & J^{\prime} & 2 \\
K & -K^{\prime} & 0
\end{array}\right)^{2} \sum_{l_{i} l_{f}}\left(2 l_{i}+1\right)\left(2 l_{f}+1\right)\left(\begin{array}{ccc}
l_{i} & l_{f} & 2 \\
0 & 0 & 0
\end{array}\right)^{2} \cdot\left|M_{l_{i} l_{f}}^{-3}\right|^{2}
$$


Note again the presence of the factor $\delta\left(K, K^{\prime}\right)$, which implies that the quadrupolar interaction cannot induce rotational transitions with $|\Delta K| \neq 0$. The electron quadrupole radial matrix elements $M_{l_{i} l_{f}}^{-3}$ can be calculated using various formulas and recurrence relations..$^{911}$ There are, for $\lambda=2$, two types of matrix elements, those for which $l_{i}-l_{f}$ $= \pm 2$ and those for which $l_{i}=l_{f}$. They are connected through the relation ${ }^{9,11}$

$y M_{l l}^{-3}=y_{1} M_{l, l+2}^{-3}+y_{2} M_{l-1, l+1}^{-3}+y_{3} M_{l+2, l}^{-3}+y_{4} M_{l+1, l-1}^{-3}$,

with

$$
\begin{aligned}
& y=\frac{1}{3} l(l+1)\left(\eta_{f}^{2}-\eta_{i}^{2}\right), \\
& y_{1}=-\eta_{i}^{2}\left|l+1+i \eta_{f}\right|\left|l+2+i \eta_{f}\right|, \\
& y_{2}=\eta_{i} \eta_{f}[(2 l+3) /(2 l+1)]\left|l+i \eta_{i}\right|\left|l+1+i \eta_{f}\right|, \\
& y_{3}=\eta_{f}^{2}\left|l+1+i \eta_{i}\right|\left|l+2+i \eta_{i}\right|, \\
& y_{4}=-\eta_{i} \eta_{f}[(2 l+3) /(2 l+1)]\left|l+i \eta_{f}\right|\left|l+1+i \eta_{i}\right| .
\end{aligned}
$$

For the matrix elements with $\left|l_{i}-l_{f}\right|=2$, two of them are connected with two monopole matrix elements $(\lambda=0)$ by $^{9}$

$$
y_{1} M_{l+1, l+3}^{-3}+y_{2} M_{l, l+2}^{-3}=y_{3} M_{l+1}^{-1}, l+1+y_{4} M_{l l}^{-1},
$$

with

$$
\begin{aligned}
& y_{1}=4(l+1) \eta_{i}^{2}\left|l+2+i \eta_{f}\right|\left|l+3+i \eta_{f}\right|, \\
& y_{2}=-4(l+1) \eta_{i} \eta_{f}\left|l+2+i \eta_{f}\right|\left|l+1+i \eta_{i}\right|, \\
& y_{3}=\left(k_{i}^{2}-k_{f}^{2}\right)\left[2 \eta_{i}^{2} \eta_{f}^{2}+\eta_{f}^{2}(l+1)(2 l+3)-\eta_{i}^{2}(l+1)\right], \\
& y_{4}=-\left(k_{i}^{2}-k_{f}^{2}\right) 2 \eta_{i} \eta_{f}\left|l+1+i \eta_{i}\right|\left|l+1+i \eta_{f}\right| .
\end{aligned}
$$

By repeated use of Eq. (35), we obtain the recurrence relation

$$
M_{l, l+2}^{-3}=f(l)\left(\frac{M_{l^{\prime}, l^{\prime}+2}^{-3}}{f\left(l^{\prime}\right)}+\sum_{j=l}^{l-1} \frac{A(j)}{f(j+1)}\right),
$$

with $l^{\prime}<l$ and

$$
\begin{aligned}
f(l)= & \left(\eta_{f} / \eta_{i}\right)^{l}\left|\Gamma\left(l+1+i \eta_{i}\right) / \Gamma\left(l+3+i \eta_{f}\right)\right| \\
A(l)= & \frac{k_{i}^{2}-k_{f}^{2}}{4(l+1)\left|l+2+i \eta_{f}\right|\left|l+3+i \eta_{f}\right|} \frac{1}{\eta_{i}^{2}} \\
& \times\left\{\left[2 \eta_{i}^{2} \eta_{f}^{2}+\eta_{f}^{2}(l+1)(2 l+3)-\eta_{i}^{2}(l+1)\right] M_{l+1, l+1}^{-1}\right. \\
& \left.-2 \eta_{i} \eta_{f}\left|l+1+i \eta_{i}\right|\left|l+1+i \eta_{f}\right| M_{l l}^{-1}\right\}
\end{aligned}
$$

In the $\lambda=2$ case, we need only to calculate the two quadrupole radial matrix elements $M_{02}^{-3}$ and $M_{20}^{-3}$ and the monopole matrix elements $M_{l l}^{-1}$. All the other matrix elements $M_{l l^{\prime}}^{-3}$ can be generated by using the relations (36) and (32). The monopole $(\lambda=0)$ matrix elements satisfy the three-term relation ${ }^{9}$

$$
y_{1} M_{l+1, l+1}^{-1}+y_{2} M_{l l}^{-1}+y_{3} M_{l-1, l-1}^{-1}=0,
$$

with

$$
\begin{aligned}
& y_{1}=2 l\left|l+1+i \eta_{i}\right|\left|l+1+i \eta_{f}\right|, \\
& y_{2}=-(2 l+1)\left\{\left[\left(\eta_{i}^{2}+\eta_{f}^{2}\right) / \eta_{i} \eta_{f}\right] l(l+1)+2 \eta_{i} \eta_{f}\right\}, \\
& y_{3}=(2 l+2)\left|l+i \eta_{i}\right|\left|l+i \eta_{f}\right| .
\end{aligned}
$$

The first two monopole matrix elements $M_{00}^{-1}$ and $M_{11}^{-1}$ are most easily calculated by the following direct formula ${ }^{9}$ :

$$
\begin{aligned}
M_{i l}^{-1}= & \left(k_{i}-k_{f}\right)^{-2}\left(\frac{\xi}{\eta_{i}+\eta_{f}}\right)^{i\left(\eta_{i}+\eta_{f}\right)} \\
& \times \frac{\left|\Gamma\left(l+1+i \eta_{i}\right)\right|\left|\Gamma\left(l+1+i \eta_{f}\right)\right|}{(2 l+1) !} \\
& \times e^{-(\pi / 2) \xi}\left(-\chi_{0}\right)^{l} F\left(l+1-i \eta_{i}, l+1-i \eta_{f}, 2 l+2 ; \chi_{0}\right),
\end{aligned}
$$

where

$$
\chi_{0}=-4 \eta_{i} \eta_{f} / \xi^{2} .
$$

In the scattering of molecular ions by slow electrons an approximate but useful analytical formula can be derived following Stabler. ${ }^{1}$ For symmetrictop molecular ions, we obtain

$$
\begin{aligned}
\sigma_{20}\left(J K \rightarrow J^{\prime} K^{\prime}\right)= & \delta\left(K, K^{\prime}\right) \frac{64\left(\ln 2-\frac{2}{3}\right) \pi^{3} Q^{2}}{15 k_{i}^{2}} \\
& \times\left(2 J^{\prime}+1\right)\left(\begin{array}{ccc}
J & J^{\prime} & 2 \\
K & -K^{\prime} & 0
\end{array}\right)^{2}
\end{aligned}
$$

or

$$
\sigma_{20}\left(J K \rightarrow J^{\prime} K^{\prime}\right)=\delta\left(K, K^{\prime}\right) \frac{32\left(\ln 2-\frac{2}{3}\right) \pi^{3} Q^{2}}{5 k_{i}^{2}} \eta(\Delta J, K),
$$

with

$$
\begin{aligned}
\eta & =\frac{(J+K+2)(J+K+1)(J-K+2)(J-K+1)}{(2 J+3)(2 J+1)(J+2)(J+1)} \\
& \text { if } \Delta J \equiv J^{\prime}-J=+2, \\
& =2 K^{2} \frac{(J+K+1)(J-K+1)}{(J+2)(J+1) J(2 J+1)} \text { if } \Delta J=+1, \\
& =2 K^{2} \frac{(J+K)(J-K)}{(J+1)(2 J+1) J(J-1)} \text { if } \Delta J=-1, \\
& =\frac{(J+K)(J+K-1)(J-K)(J-K-1)}{(2 J+1)(2 J-1) J(J-1)} \text { if } \Delta J=-2 .
\end{aligned}
$$

When $K \neq 0$, the quadrupolar interaction induces both the $|\Delta J|=2$ transitions and the $|\Delta J|=1$ transitions. Depending upon the magnitude of the ratio $(\mu / Q)^{2}$ and the energy range, these $\sigma_{20}$ with $|\Delta J|=1$ and $K \neq 0$ may be smaller, comparable, or greater than $\sigma_{10}$. In the special case of vanishing dipole moment $(\mu=0)$, the $\sigma_{20}$ with $K \neq 0$ provides the 
major contributions to the $|\Delta J|=1$ transitions.

Equation (43) is analogous to the threshold formula Eq. (30) for $\sigma_{10}$. The exact Coulomb-Born $\sigma_{20}$ should depend upon not only $k_{i}^{-2}$ but also an increasing function of energy like $f_{E 1}$ in Eq. (28). Unfortunately, this additional energy-dependent function cannot be obtained in simple analytic form in the case of quadrupole transition. The range of validity of the approximate formula Eq. (43) can be obtained following Stabler's discussion. ${ }^{1}$ The corresponding range of validity of the exact Coulomb-Born formula [Eq. (28)] for $\sigma_{10}$ is much wider, and this dipolar formula is expected to work well as long as $k^{2}<1$ a.u. More refined theory like close-coupling for fixed-nuclei approxi- mation is desirable for testing the validity of the present theory in the high-energy range.

\section{ROTATIONAL EXCITATION INDUCED BY THE HIGHER MULTIPOLAR INTERACTIONS}

The rotational excitation cross sections $\sigma_{\lambda \nu}$ for $\lambda \geqslant 3$ and $\nu=0$ are usually much smaller than the dipolar $\sigma_{10}$ and quadrupolar $\sigma_{20}$ terms and of small significance in partical applications. However, it is worth noting that the leading term that induces rotational transitions around the symmetry axis $(\Delta K \neq 0)$ is due to the potential $v_{3, \pm 3}(r)$. In this case,

$$
\sigma_{3, \pm 3}\left(J K \rightarrow J^{\prime} K^{\prime}\right)=4\left(\frac{k_{f}}{k_{i}}\right)\left(M_{3, \pm 3}\right)^{2}\left(2 J^{\prime}+1\right)\left(\begin{array}{ccc}
J & J^{\prime} & 3 \\
K & -K^{\prime} & \mp 3
\end{array}\right)^{2} \sum_{l_{i} l_{f}}\left(2 l_{i}+1\right)\left(2 l_{f}+1\right)\left(\begin{array}{ccc}
l_{i} & l_{f} & 3 \\
0 & 0 & 0
\end{array}\right)^{2}\left|M_{l_{i} l_{f}}^{-4}\right|^{2},
$$

with

$$
\Delta K=K^{\prime}-K= \pm 3 .
$$

To calculate $\sigma_{3, \pm 3}$, we need the components of the octupole tensor $M_{3, \pm 3}$ and to evaluate the more complicated octupole radial matrix elements $M_{l_{i} l_{f}}^{-4}$. The $\sigma_{3, \pm 3}$ cross sections are usually much smaller than $\sigma_{10}$ or $\sigma_{20}$ and we shall not consider them further.

\section{A FumERICAL EXAMPLE: THE ROTATIONAL EXCITATION OF THE $\mathrm{H}_{3} \mathrm{O}^{+}$ION}

A. Dipole moment of the $\mathrm{H}_{3} \mathrm{O}^{+}$ion

There are no experimental data on the dipole moment of the $\mathrm{H}_{3} \mathrm{O}^{+}$ion. Information of the $\mathrm{H}_{3} \mathrm{O}^{+}$ion is available from measurements in the solid phase $\mathrm{e}^{12,18}$ and in the solution. ${ }^{19}$ The gas-phase data are relatively rare. ${ }^{20}$ Theoretical studies show that the equilibrium structure of the $\mathrm{H}_{3} \mathrm{O}^{+}$ ion is either planar ${ }^{13,14,21}$ or pyramid pr,22,23 $^{15}$ ith a very small inversion barrier of the order of magnitude of $0.1-0.3 \mathrm{kcal} / \mathrm{mole}$. The only exception is due to the work of Lischka and Dyczmons, ${ }^{24}$ who found that the inversion varrier could be greatly increased by the inclusion of the correlation ener- gy. It appears, therefore, that no decisive conclusion can be drawn about the conformation of this ion at the present time. In this study, we have considered two geometries of the $\mathrm{H}_{3} \mathrm{O}^{+}$ion, the planar one with $\theta_{\mathrm{HOH}}=120^{\circ}$ and vanishing dipole moment and a pyramid with $\theta_{\mathrm{HOH}}=118.1^{\circ}$ and $\mu=0.22$ a.u. ${ }^{14}$ The latter value reflects the typical dipole moment for a flat pyramid, which is borne out by most theoretical calculations. Thus, the results presented below should provide an estimate of the efficiencies of the rotational excitation processes that is of the correct order of magnitude. It should be noted, however, that the dipole-induced rotational excitation cross sections are directly proportional to the square of the dipole moment. Therefore a change in the magnitude of the dipole moment simply scales the results by a constant.

\section{B. Quadrupole moment of the $\mathrm{H}_{3} \mathrm{O}^{+}$ion}

The quadrupole moment $Q$ of the $\mathrm{H}_{3} \mathrm{O}^{+}$ion about the center of mass can be estimated using a pointcharge model and the charge population analys is performed by Grahn. ${ }^{13}$ We consider again the two geometries described in Sec. VIA. The results are summarized in Table I.

TABLE I. Electric dipole and quadrupole moments, and the rotational constants of $\mathrm{H}_{3} \mathrm{O}^{+}{ }^{\mathrm{a}}$

\begin{tabular}{lllcccccc}
\hline \hline Geometry & $d_{\mathrm{OH}}$ & \multicolumn{1}{c}{$h^{\mathrm{b}}$} & \multicolumn{1}{c}{$\theta$} & \multicolumn{1}{c}{$\beta$} & $\mu$ & $Q$ & $A$ & $B$ \\
\hline Planar & 1.8 & 0 & $120^{\circ}$ & $90^{\circ}$ & 0 & -2.212 & $2.801 \times 10^{-5}$ & $5.602 \times 10^{-5}$ \\
Pyramid & $1.8^{\mathrm{c}}$ & $0.25^{\mathrm{c}}$ & $118.1^{\circ}$ & $82.05^{\circ}$ & $0.22^{\mathrm{c}}$ & -2.214 & $2.854 \times 10^{-5}$ & $5.552 \times 10^{-5}$ \\
\hline \hline
\end{tabular}

${ }^{a}$ Angles are measured in degrees and the other quantities are measured in atomic units.

${ }^{\mathrm{b}} h=d_{\mathrm{OH}} \cos \beta$ is a measurement of the nonplanarity of the $\mathrm{H}_{3} \mathrm{O}^{+}$ion.

${ }^{\mathrm{c}}$ The theoretical values taken from Ref. 14 . 


\section{Rotational constant of the $\mathrm{H}_{3} \mathrm{O}^{+}$ion}

The moment of inertia of pyramidal molecules $X_{3}$ can be calculated by the following expressions:

$I_{x}=I_{y}=2 m_{Y} d_{X Y}^{2} \sin ^{2} \frac{1}{2} \theta+\left(m_{X} m_{Y} / M\right) d_{X Y}^{2}\left(3-4 \sin ^{2} \frac{1}{2} \theta\right)$,

$$
I_{z}=4 m_{Y} d_{X Y}^{2} \sin ^{2} \frac{1}{2} \theta
$$

where the $z$ axis is the $C_{3}$ symmetry axis and $\theta$ is the $Y-X-Y$ bond angle. The $m_{X}, m_{Y}$, and $M$ are the masses of atoms $X, Y$, and the molecule $X Y_{3}$, respectively, and $d_{X Y}$ is the $X-Y$ bond distance. The acute angle $\beta$ between the $X-Y$ bond and the symmetry axis is related to the bond angle $\theta$ by

$$
\sin \frac{1}{2} \theta=\frac{1}{2} \sqrt{3} \sin \beta
$$

The rotational constants $A \equiv \hbar^{2} / 2 I_{z}$ and $B \equiv \hbar^{2} / 2 I_{x}$ $=\hbar^{2} / 2 I_{y}$ were calculated using (46a) and (46b) and the results are given in Table $\mathrm{I}$. The derived rotational constants $A$ and $B$ are rather close to the values of the corresponding isoelectronic molecule $\mathrm{NH}_{3}$. (For $\mathrm{NH}_{3}, A=2.87 \times 10^{-5}$ and $B$ $=4.53 \times 10^{-5}$ a.u. ${ }^{16}$ )

\section{Numerical results and discussion}

Consider first the planar geometry of the $\mathrm{H}_{3} \mathrm{O}^{+}$ ion. The permanent dipole moment $\mu$ is zero and $\sigma_{10} \equiv 0$. The leading contribution to the rotational transitions arises from the quadrupolar $\sigma_{20}$ term. Table I shows that the derived quadrupole moment and the rotational constants of $\mathrm{H}_{3} \mathrm{O}^{+}$of the planar geometry are not very different from those for the flat pyramid. Thus, the $\sigma_{20}$ calculated for the two geometries will differ only by a few percent. Accordingly, we present the results for the pyramid geometry only.

The rotational excitation cross sections for the process $^{25}$

$$
e^{-}+\mathrm{H}_{3} \mathrm{O}^{+}(J=5, K=0) \rightarrow e^{-}+\mathrm{H}_{3} \mathrm{O}^{+}\left(J^{\prime}=6, K^{\prime}=0\right)
$$

were calculated using Eqs. (28) and (30). Only the dipole $\sigma_{10}$ term contributes to the transition. The quadrupole $\sigma_{20}$ term is identically zero. The energy-dependent behavior of $\sigma_{10}$ is depicted in Fig. 2 . The cross section $\sigma_{10}$ is largest at excitation threshold and decreases with increasing energy. The behavior of the function $f_{E 1}$ is also shown in the figure. The function $f_{E 1}$ is an increasing function of energy. In the low-energy region, however, $f_{E 1}$ can also be easily estimated by using the following expression ${ }^{5}$ :

$$
f_{E 1}^{\prime}(\xi<0) \simeq\left(32 \pi^{3} / 9 \sqrt{3}\right)\left(1+0.218|\xi|^{-2 / 3}\right)
$$

In Fig. 2, the energy-dependent behavior of $f_{E 1}$ and $f_{E 1}^{\prime}$ is compared. It is seen that $f_{E 1}^{\prime}$ is an adequate representation for $E \varsigma 0.2 \mathrm{eV}$, the latter being

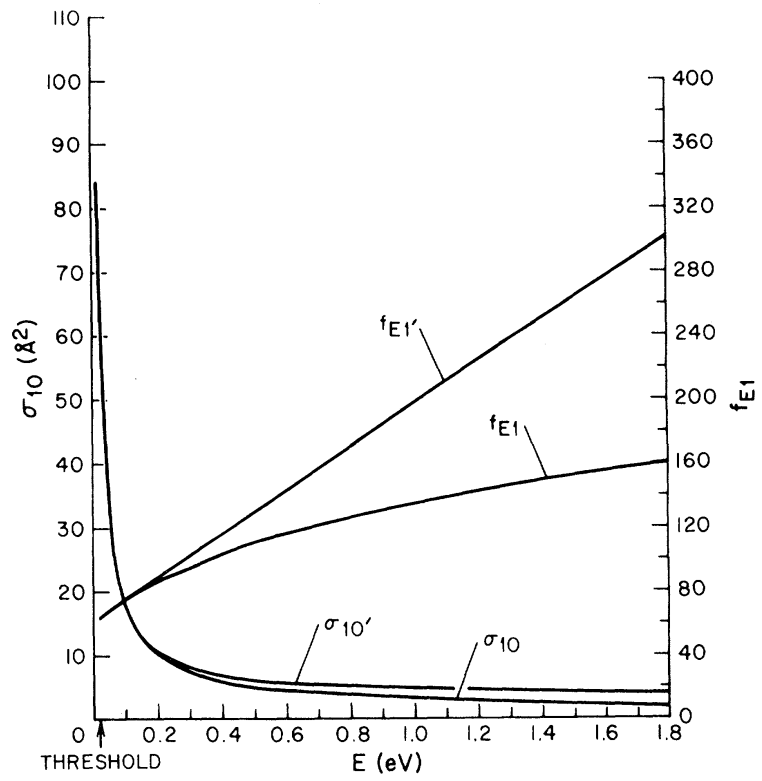

FIG. 2. Calculated results of excitation $(J=5, K=0$ $\left.\rightarrow J^{\prime}=6, K^{\prime}=0\right)$ cross sections $\sigma_{10}$ and $\sigma_{10}^{\prime}$ due to $v_{10}$. Also shown are the $E 1$ Coulomb excitation functions $f_{E 1}$ and $f_{E 1}^{\prime}$.

about 10 times the threshold energy. The corresponding cross sections $\left(\sigma_{10}^{\prime}\right)$ calculated by using $f_{E 1}^{\prime}$ are also depicted in Fig. 2 for comparison.

Figure 3 shows the energy-dependent excitation cross sections for the process ${ }^{25}$

$$
e^{-}+\mathrm{H}_{3} \mathrm{O}^{+}(J=5, K=1) \rightarrow e^{-}+\mathrm{H}_{3} \mathrm{O}^{+}\left(J^{\prime}=6, K^{\prime}=1\right) .
$$

Both $\sigma_{10}$ and $\sigma_{20}$ contribute to the transition. The quadrupole $\sigma_{20}$ terms were calculated using the approximate formulas Eqs. (43) and (44). Because of the smallness of the ratio $(\mu / Q)^{2} \sim 10^{-2}$, the ratio of $\sigma_{10}$ to $\sigma_{20}$

$$
\frac{\sigma_{10}}{\sigma_{20}}=\left(\frac{\mu}{Q}\right)^{2} \frac{J(J+2)}{K^{2}} \frac{15}{128 \pi^{4}\left(\ln 2-\frac{2}{3}\right)} f_{E_{1}}
$$

is less than unity in the lower-energy regions $E \lesssim 0.6 \mathrm{eV}$. However, because $f_{E 1}$ is an increasing function of energy, the ratio becomes greater than unity for energies higher than $0.6 \mathrm{eV}$. Thus, both $\sigma_{10}$ and $\sigma_{20}$ make important contributions to the specific excitation process. However, it should be remarked here that $\sigma_{20}$ predicted by the approximate formula Eq. (43) decreases a little faster with energy than the exact Coulomb-Born cross section in the high-energy region (cf. Sec. IV). Thus, the crossing of the $\sigma_{20}$ and $\sigma_{10}$ curves is probably spurious and is an indication that the approximate $\sigma_{20}$ becomes less reliable for energy higher than the crossing point.

The excitation cross sections for the processes ${ }^{25}$

$e^{-}+\mathrm{H}_{3} \mathrm{O}^{+}(J=5,1<K \leqslant 5) \rightarrow e^{-}+\mathrm{H}_{3} \mathrm{O}^{+}\left(J^{\prime}=6,1<K^{\prime} \leqslant 5\right)$ 


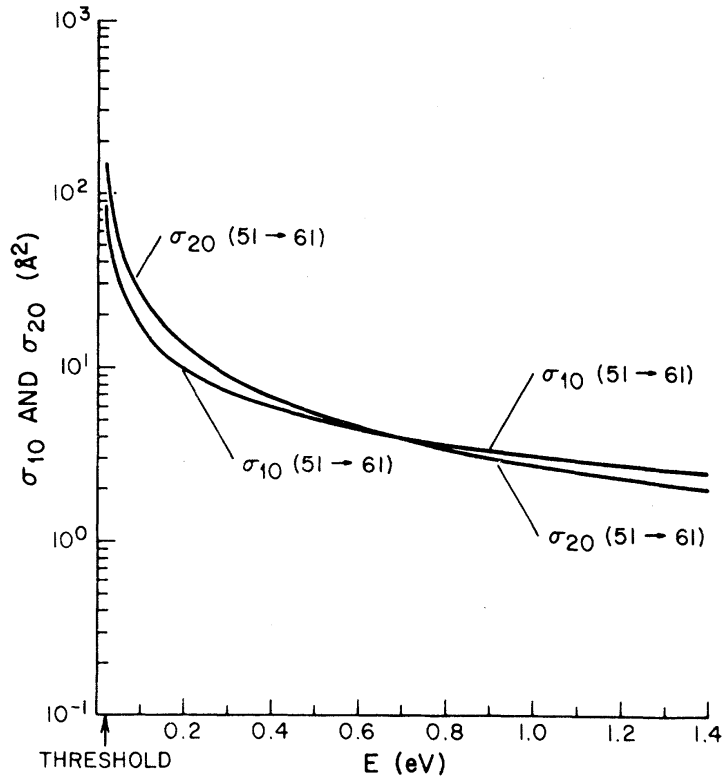

FIG. 3. Calculated results of excitation $(J=5, K=1$ $\left.\rightarrow J^{\prime}=6, K^{\prime}=1\right)$ cross sections due to $v_{10}$ and $v_{20}$.

can be evaluated by scaling the results of Fig. 3 . The function $f_{E 1}$ is independent of $K$ and the only dependence of $K$ arises from the geometric factors $g_{ \pm}$in $\sigma_{10}$ and $\eta(\Delta J, K)$ in $\sigma_{20}$. Two examples corresponding to the $\left(J=5, K=2 \rightarrow J^{\prime}=6, K^{\prime}=2\right)$ and $(J=5$, $\left.K=5 \rightarrow J^{\prime}=6, K^{\prime}=5\right)$ transitions are shown in Fig. 4. It is noted that the ratio $\sigma_{10} / \sigma_{20}$ decreases with increasing $K$ and $\sigma_{20}$ becomes the dominant term when $K \gtrsim 2$.

\section{DISCUSSION AND CONCLUSION}

The accuracy of the present theory is based on the validity of the following three assumptions: (i) the long-range asymptotic form of the interaction potential can be used throughout the whole range of electron-molecular ion separation, (ii) the distortion of the electron wave function is small such that the Coulomb waves can be used for the initial and the final states of the scattered electron, and (iii) the first-order perturbation theory is applicable.

To examine the validity of these assumptions, we use the partial-wave-expansion form of the cross section $\sigma_{\lambda \nu}$ in Eq. (25). We shall discuss especially the dipole-transition cross sections. For $\sigma_{10}$, the summations over $l_{i}$ and $l_{f}$ can be rewritten as

$$
\begin{aligned}
b_{0}^{E_{1}} & \equiv \sum_{l_{i} l_{f}}\left(2 l_{i}+1\right)\left(2 l_{f}+1\right)\left(\begin{array}{ccc}
l_{i} & l_{f} & 1 \\
0 & 0 & 0
\end{array}\right)^{2}\left|M_{l_{i} l_{f}}^{-2}\right|^{2} \\
& =\sum_{l=0}^{\infty}\left[l\left|M_{l-1, l}^{-2}\right|^{2}+(l+1)\left|M_{l+1, l}^{-2}\right|^{2}\right] .
\end{aligned}
$$

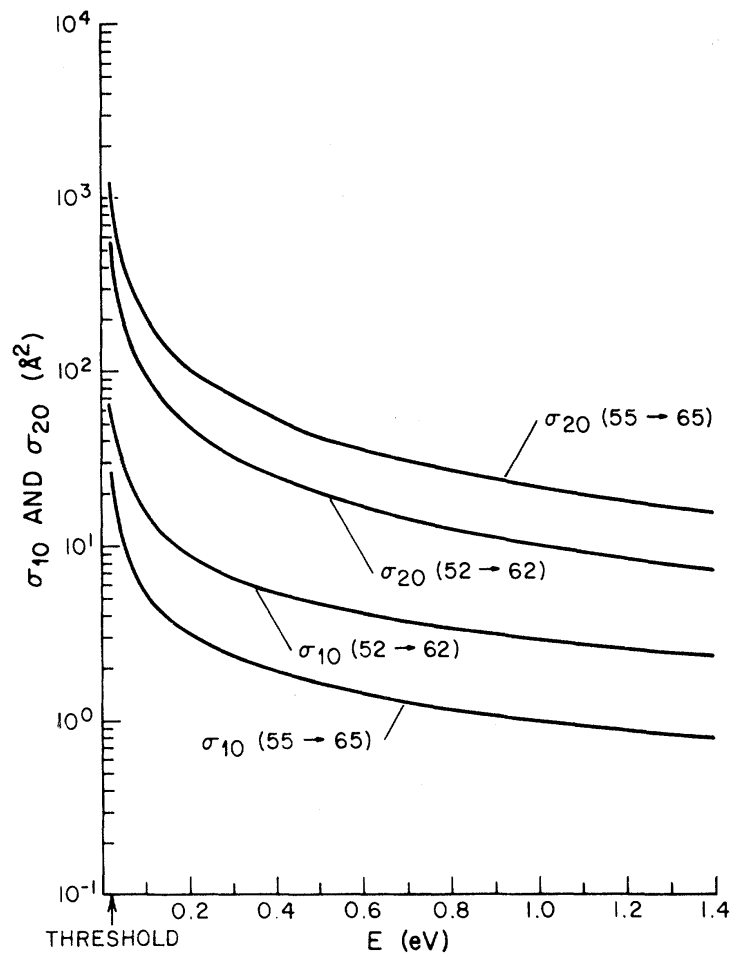

FIG. 4. Excitation cross sections $\sigma_{10}$ and $\sigma_{20}$ corresponding to $\left(J=5, K=2 \rightarrow J^{\prime}=6, K^{\prime}=2\right)$ and $(J=5, K=5$ $\left.\rightarrow J^{\prime}=6, K^{\prime}=5\right)$ transitions.

The sum over $l$ can be put in a more tractable form by reducing the dipole elements $M_{l \pm 1, l}^{-2}$ in terms of the monopole matrix elements $M_{l, l}^{-1} \cdot{ }^{9}$ The result is

$$
\begin{aligned}
b_{0}^{E 1}=\sum_{l=1}^{\infty} l & {\left[\left(k_{i}^{2}+k_{f}^{2}+2 k_{i}^{2} \eta_{i}^{2} / l\right)\left(\left|M_{l-1, l-1}^{-1}\right|^{2}+\left|M_{l, l}^{-1}\right|^{2}\right)\right.} \\
& \left.\quad-4 k_{i} k_{f}\left|1+i \eta_{i} / l\right|\left|1+i \eta_{f} / l\right| M_{l-1, l-1}^{-1} M_{l l}^{-1}\right] .
\end{aligned}
$$

Using the recurrence relations for $M_{l l}^{-1}$ [Eqs. (39) and (40)] and defining the function $Q(l)$

$$
\begin{aligned}
l Q(l) \equiv & 2 k_{i} k_{f}\left[\left(l^{2}+\eta_{i}^{2}\right)\left(l^{2}+\eta_{f}^{2}\right)\right]^{1 / 2} M_{l l}^{-1} M_{l-1, l-1}^{-1} \\
& -\left[\left(k_{i}^{2}+k_{f}^{2}\right) l^{2}+2 k_{i}^{2} \eta_{i}^{2}\right]\left|M_{l-1, l-1}^{-1}\right|^{2},
\end{aligned}
$$

it can be readily shown that

$$
b_{0}^{E 1}=\sum_{l=1}^{\infty}[Q(l+1)-Q(l)]
$$

This of course leads to the formula

$$
b_{0}^{E_{1}}=-Q(1) \text {. }
$$

The proof of relations (52) and (53) was first provided by Biedenharn. ${ }^{26,27}$ Since $Q(1)$ (and therefore $\sigma_{10}$ ) depends only upon the two monopole matrix elements $M_{00}^{-1}$ and $M_{11}^{-1}$, it provides us a sim- 
ple way to examine the validity of our assumptions.

The monopole radial matrix elements $M_{00}^{-1}$ and $M_{11}^{-1}$ will be evaluated as a function of $R$, the upper limit of the radial integration,

$$
M_{l l}^{-1}(R) \equiv \frac{1}{k_{i} k_{f}} \int_{0}^{R} F_{l}\left(k_{i} r\right) r^{-1} F_{l}\left(k_{f} r\right) d r .
$$

The Coulomb wave functions $F_{l}(k r)$ are obtained by using the methods described by Fröberg ${ }^{28}$ and are calculated to the accuracy of eight digits. An example which corresponds to the rotational transition of $\mathrm{H}_{3} \mathrm{O}^{+}\left(J=5, K=0 \rightarrow J^{\prime}=6, K^{\prime}=0\right)$ at $k_{i}=0.1$ a.u. is given in Table II, where $M_{l l}^{-1}(R=\infty)$ is calculable by using Eq. (41) directly. It is noted that about $11.8 \%$ of $M_{00}^{-1}(R=\infty)$ and $6.6 \%$ of $M_{11}^{-1}$ $(R=\infty)$ comes from the inner region $R \leqslant 2 a_{0}$, and more than $60 \%$ of both $M_{00}^{-1}(R=\infty)$ and $M_{11}^{-1}(R=\infty)$ is due to $R>20 a_{0}$. Even for $R$ as large as $50 a_{0}$, there still comes some $40 \%$ of contribution from the longer range of $R$ ( $\left.R \geqslant 50 a_{0}\right)$. Thus, it is apparent that the dominant contribution to the rotational excitation is caused by the long-range interaction. Our assumption (i), that the long-range asymptotic form of the interaction potential can be used throughout the whole range of $r$ is, therefore, justified with an error of about $10 \%$ introduced by the unknown short-range forces. Assumptions (ii) and (iii) are also justified because the dominant contributions come from $R \geqslant 20 a_{0}$. At these long distances, the dipole interaction energy is much smaller than the kinetic energy of the electron. Thus, the distortion of the electron wave function is small and the first-order perturbation treatment is adequate.

In conclusion, our assumptions for the lowenergy rotational excitations of molecular ions seems justified. Because of the strong Coulomb acceleration, the short-range interaction does have some (though small) contribution to the in-

TABLE II. Ratio of monopole radial matrix elements $M_{00}^{-1}(R) / M_{00}^{-1}(R=\infty)$ and $M_{11}^{-1}(R) / M_{11}^{-1}(R=\infty)$ corresponding to the rotational transition $\left(J=5, K=0 \rightarrow J^{\prime}=6, K^{\prime}=0\right)$ of $\mathrm{H}_{3} \mathrm{O}^{+}$at $k_{i}=0.1$ a.u.

\begin{tabular}{ccc}
\hline \hline$R\left(a_{0}\right)$ & $M_{00}^{-1}(R) / M_{00}^{-1}(R=\infty)$ & $M_{11}^{-1}(R) / M_{11}^{-1}(R=\infty)$ \\
\hline 1 & 0.1006 & 0.0098 \\
2 & 0.1176 & 0.0663 \\
3 & 0.1378 & 0.1361 \\
4 & 0.1828 & 0.1725 \\
5 & 0.2118 & 0.1783 \\
10 & 0.2894 & 0.2820 \\
20 & 0.4034 & 0.3882 \\
30 & 0.4895 & 0.4801 \\
40 & 0.5555 & 0.5553 \\
50 & 0.5968 & 0.5981 \\
$\infty$ & 1.0000 & 1.0000 \\
\hline \hline
\end{tabular}

elastic process. For high-energy collisions, more realistic potential (i.e. including the short-range part) and more refined scattering theory are required to assess the validity of the present theory.

\section{ACKNOWLEDGMENTS}

This research was supported by the National Science Foundation under contract No. GP-39308X. The author wishes to thank Professor Gordon Dunn for suggesting this work and for discussions. $\mathrm{He}$ is also indebted to Professor Alexander Dalgarno and Professor William Reinhardt for critical reading and suggestions on the manuscript, and to Dr. Edward Chang for stimulating discussions on Sec. VII.

\section{APPENDIX: STABLER'S THEORY OF COULOMB EXCITATION OF MOLECULAR IONS BY ELECTRON IMPACT}

In this appendix we demonstrate that Stabler's theory is not applicable to the electron-dipolar molecular-ion scattering system. For simplicity, we assume that the dipolar molecular ion is a linear rigid rotor. Then all the formulas in Refs. 4 and 5 are applicable.

Consider the process

$$
e^{-}+A B^{+}(j m) \rightarrow e^{-}+A B^{+}\left(j^{\prime} m^{\prime}\right),
$$

where $j$ and $m$ are, respectively, the rotational and projection quantum numbers of the $A B^{+}$linear rigid-rotor ion. From Eqs. (5) and (10) of Ref. 5, the transition matrix element $A_{f i}$ for the process (A1) can be written as

$$
\begin{aligned}
A_{f i}=-\sum_{\lambda=1}^{\infty} Q_{\lambda} \int d \hat{S} Y_{j^{\prime} m^{\prime}}^{*}(\hat{s}) Y_{j m}(\hat{s}) \\
\\
\times\left\langle\overrightarrow{\mathrm{k}}_{f}\left|r^{-\lambda-1} P_{\lambda}(\hat{r} \cdot \hat{s})\right| \overrightarrow{\mathrm{k}}_{i}\right\rangle,
\end{aligned}
$$

where $Q_{\lambda}$ is the $\lambda$ th electron moment of the charge distribution of the molecular ion. Thus $Q_{1}$ is the dipole moment and $Q_{2}$ the quadrupole moment. In Eq. (A2), $\hat{r}$ is the coordinate of the incident electron relative to the center of mass of the molecule, $\hat{s}$ denotes the internuclear coordinate of the molecular ion, and the spherical harmonic $Y_{j m}$ represents the molecular rotator in the quantum states $(j, m)$, etc.

Stabler assumed that the Coulomb wave functions can be approximated by ${ }^{1}$

$$
\left|\overrightarrow{\mathrm{k}}_{i}\right\rangle \cong\left(\pi / k_{i} r\right)^{1 / 2} \sum_{l=0}^{\infty}(2 l+1) J_{2 l+1}(\sqrt{8 r}) P_{l}\left(\hat{k}_{i} \cdot \hat{r}\right)
$$

and

$$
\left|\overrightarrow{\mathrm{k}}_{f}\right\rangle \cong\left(\pi / k_{f} r\right)^{1 / 2} \sum_{l^{\prime}=0}^{\infty}\left(2 l^{\prime}+1\right) J_{2 l^{\prime}+1}(\sqrt{8 r}) P_{l^{\prime}}\left(\hat{k}_{f} \cdot \hat{r}\right) .
$$

Substituting (A3) into (A2), we get 


$$
A_{f i}=-\frac{\pi}{\left(k_{i} k_{f}\right)^{1 / 2}} \sum_{\lambda=1}^{\infty} Q_{\lambda} \sum_{l l^{\prime}}(2 l+1)\left(2 l^{\prime}+1\right) I_{R} I_{A}
$$

where $I_{R}$ is the radial integral

$$
I_{R}=\int_{0}^{\infty} \frac{d r}{r} J_{2 l^{\prime}+1}(\sqrt{8 r}) J_{2 l+1}(\sqrt{8 r})
$$

and $I_{A}$ is the angular integral

$$
\begin{aligned}
I_{A}=\int & d \hat{r} d \hat{s} P_{l^{\prime}}\left(\hat{k}_{f} \cdot \hat{r}\right) P_{\lambda}(\hat{r} \cdot \hat{s}) \\
& \times P_{l}\left(\hat{k}_{i} \cdot \hat{r}\right) Y_{j^{\prime} m^{\prime}}^{*}(\hat{s}) Y_{j m}(\hat{s}) .
\end{aligned}
$$

The radial integral can be evaluated using the formula ${ }^{17}$

$$
\int_{0}^{\infty} t^{-\lambda} J_{\mu}(a t) J_{\nu}(a t) d t=\frac{\left(\frac{1}{2} a\right)^{\lambda-1} \Gamma(\lambda) \Gamma\left(\frac{1}{2} \mu+\frac{1}{2} \nu-\frac{1}{2} \lambda+\frac{1}{2}\right)}{2 \Gamma\left(\frac{1}{2} \lambda+\frac{1}{2} \nu-\frac{1}{2} \mu+\frac{1}{2}\right) \Gamma\left(\frac{1}{2} \lambda+\frac{1}{2} \mu-\frac{1}{2} \nu+\frac{1}{2}\right) \Gamma\left(\frac{1}{2} \lambda+\frac{1}{2} \mu+\frac{1}{2} \nu+\frac{1}{2}\right)},
$$

if $\operatorname{Re}(\mu+\nu+1)>\operatorname{Re}(\lambda)>0$. Thus, $I_{R}$ becomes

$$
\begin{aligned}
I_{R}= & 2 \int_{0}^{\infty} t^{-1} d t J_{2 l+1}(t) J_{2 l^{\prime}+1}(t) \\
& =\frac{\Gamma\left(l^{\prime}+l+1\right)}{2 \Gamma\left(1+l^{\prime}-l\right) \Gamma\left(1+l-l^{\prime}\right) \Gamma\left(2+l+l^{\prime}\right)} .
\end{aligned}
$$

The denominator of the right-hand side of Eq. (A8) becomes infinite for integral values of $l$ and $l^{\prime}$ and $\left|l-l^{\prime}\right| \geqslant 1$. We are left with the radial selection rule that $l=l^{\prime}$. Next we evaluate the angular integral in (A6). If we consider only the $\lambda=1 \mathrm{di}-$ pole term, the integral becomes

$$
\begin{aligned}
I_{A} & \propto \int d \hat{r} Y_{l^{\prime} m_{l}}(\hat{r}) Y_{1 \nu}(\hat{r}) Y_{l m_{l}}(\hat{r}) \\
& \propto\left(\begin{array}{lll}
l^{\prime} & 1 & l \\
0 & 0 & 0
\end{array}\right) .
\end{aligned}
$$

Thus, the angular selection rule is $\left|l^{\prime}-l\right|=1$.

Therefore, within the framework of Stabler's theory, $A_{f i}$ is identically zero for the $\lambda=1$ term because the radial and angular selection rules are in conflict. Thus, Stabler's theory does not apply to the rotational excitation of dipolar ions. ${ }^{29}$
${ }^{1}$ R. C. Stabler, Phys. Rev. 131, 679 (1963).

${ }^{2}$ D. H. Sampson, Phys. Rev. 137, A4 (1965).

${ }^{3} \mathrm{E}$. Chang and A. Temkin, J. Phys. Soc. Jpn. 29, 172 (1970).

${ }^{4}$ R. F. Boikova and V. D. Obeydkov, Zh. Eksp. Teor. Fiz. 54, 1439 (1968) [Sov. Phys.-JETP 27, 772 (1968)].

${ }^{5}$ Shih-I Chu and A. Dalgarno, Phys. Rev. A 10, 788 (1974); 12, 725(E) (1975).

${ }^{6}$ D. E. Stogryn and A. P. Stogryn, Mol. Phys. 11, 371 (1.966).

7Y. Itikawa, J. Phys. Soc. Jpn. 30, 835 (1971).

${ }^{8}$ A. R. Edmonds, Angular Momentum in Quantum Mechanics (Princeton U.P., Princeton, N. J., 1960).

${ }^{9} \mathrm{~K}$. Alder, A. Bohr, T. Huus, B. Mottelson, and A. Winther, Rev. Mod. Phys. 28, 432 (1956).

${ }^{10}$ E. P. Wigner, Phys. Rev. 73, 1002 (1948).

${ }^{11}$ L. C. Biedenharn, J. L. McHale, and R. M. Tahler, Phys. Rev. 100, 376 (1955).

${ }^{12}$ R. Grahn, Ark. Fys. 19, 147 (1961.).

${ }^{13}$ R. Grahn, Ark. Fys. 21,1 (1962).

${ }^{14} \mathrm{~J}$. W. Moskowitz and $\vec{M}$. C. Harrison, J. Chem. Phys. 43, 3550 (1965).

${ }^{15}$ D. M. Bishop, J. Chem. Phys. 43, 4453 (1965).

${ }^{16} \mathrm{G}$. Herzberg, Infrared and Raman Spectra of Polyatomic
Molecules (Van Nostrand, New York, 1945).

${ }^{17} \mathrm{G}$. N. Watson, Theory of Bessel Functions, 2nd ed. (Cambridge U.P., London, 1944).

${ }^{18} \mathrm{~J}$. O. Lundgren and J. M. Williams, J. Chem. Phys. $\underline{58}$, 788 (1973).

${ }^{19}$ M. Eigen, Angew. Chem. 75, 489 (1963), and references therein.

${ }^{20}$ M. DePaz, J. J. Leventhal, and L. Friedman, J. Chem. Phys. 51, 3748 (1969); W. P. Kraemer and G. H. F. Diercksen, Chem. Phys. Lett. $\underline{5}, 463$ (1970).

${ }^{21}$ P. A. Kollman and L. C. Allen, J. Am. Chem. Soc. 92, $6101(1970)$.

${ }^{22} \mathrm{~J}$. Almlöf and U. Wahlgren, Theor. Chim. Acta 28, 161 (1973).

${ }^{23}$ B. D. Joshi, J. Chem. Phys. 47, 2793 (1967).

${ }^{24} \mathrm{H}$. Lischka and V. Dyczmons, Chem. Phys. Lett. 23 , 167 (1973).

${ }^{25}$ These are typical processes at room temperature.

${ }^{26}$ L. C. Biedenharn, Phys. Rev. 102, 262 (1956).

${ }^{27}$ R. M. Thaler, M. Goldstein, J. L. McHale, and L. C. Biedenharn, Phys. Rev. 102, 1567 (1956).

${ }^{28}$ C. E. Fröberg, Rev. Mod. Phys. 27, 399 (1955).

${ }^{29}$ The same conclusion was also reached by D. H. Sampson [Phys. Rev. 137, A4 (1965), footnote 17]. 